\title{
QUALITY IMPROVEMENT Building the business case for quality improvement: a framework for evaluating return on investment
}

\author{
Authors: Amar Shah ${ }^{A}$ and Steven Course ${ }^{B}$
}

There is increasing interest and belief in applying quality improvement (QI) to help solve our most complex challenges in healthcare, yet little published literature to help leaders develop a business case and evaluate return on investment from QI. This is even more pronounced in fields such as mental health and community health services. This paper presents a framework to help identify, understand and evaluate return on investment from large-scale application of QI in healthcare providers. The framework has been developed at East London NHS Foundation Trust (ELFT), a provider of predominantly mental health and community health services to a population of 1.5 million people, which has been undertaking QI at scale since 2014. This paper presents case studies and examples from ELFT to illustrate return on investment from QI at multiple levels: improving outcomes for patients and service users, improving the experience of staff, improving productivity and efficiency, avoiding costs, reducing costs and increasing revenue.

KEYWORDS: Quality improvement, quality, business case, organisational change

\section{Introduction}

There is an increasing recognition within healthcare that quality improvement (QI) may help take us beyond where performance management and quality assurance have helped us reach ${ }^{1}-$ going from 'good to great' as described by Jim Collins ${ }^{2}$ in 2001. At the same time, it is often a challenge for healthcare leaders and boards to articulate the return on investment from applying QI at scale, in order to create and approve a business case for this investment. ${ }^{3}$ This is partly because the task of applying QI at scale within a healthcare organisation is not just about developing a new service or creating a new project, but about developing a new operating model for the entire organisation. There is also very little published literature to turn to in helping understand the

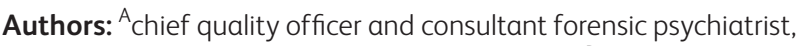
East London NHS Foundation Trust, London, UK; ${ }^{B}$ chief financial officer and deputy chief executive, East London NHS Foundation Trust, London, UK return on investment from applying QI within the diverse range of healthcare contexts - and almost nothing published in the context of mental health or community health services. ${ }^{4}$ Swensen et al's framework for evaluating return on investment from QI includes four domains of interest for healthcare providers: the needs of patients, reputation, esprit de corps, and financial return sufficient to maintain state-of-the-art medication practices. $^{3}$

Return on investment from applying QI at scale is not just about cost reduction, but about bringing new strategic capability to the organisation which can drive increased revenue, improving efficiency and productivity, and also aligning the organisation more directly to a common purpose of quality of care. ${ }^{5}$ Here we present a comprehensive framework that leaders can use to both articulate and assess the return on investment from applying QI at scale (see Fig 1). It derives from the work at East London NHS Foundation Trust (ELFT), a provider of mental health, community health, and some primary care within East London, Luton and Bedfordshire, covering a population of 1.5 million people and with a workforce of 5,000. The trust has been applying QI at scale since

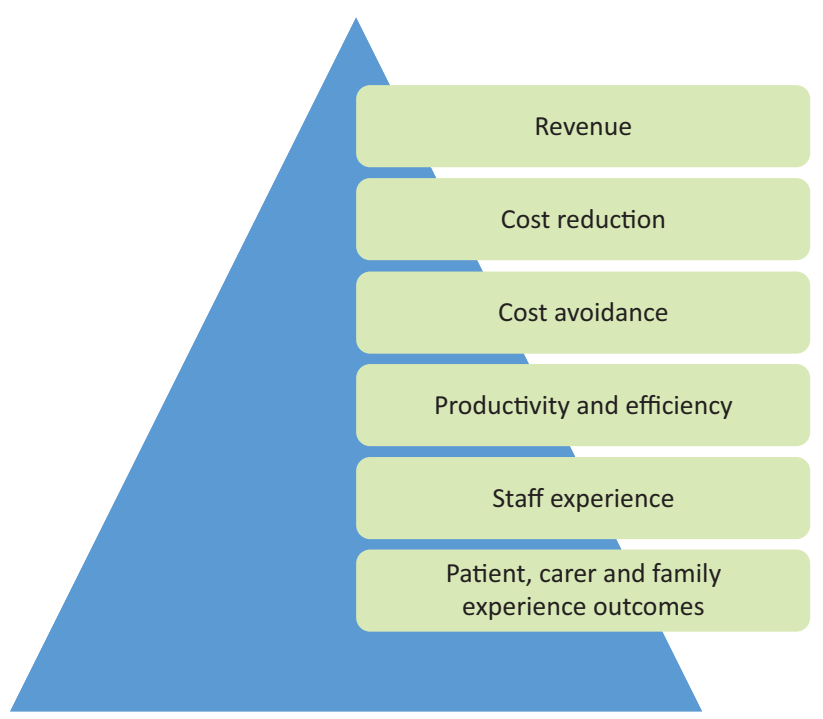

Fig 1. The ELFT framework for evaluating return on investment from quality improvement. ELFT = East London NHS Foundation Trust. 
2014 and now applies QI within every aspect of its operations across all clinical services through to corporate support services. ${ }^{6}$

Demonstrating cause and effect within the real-life complexity of a healthcare organisation is difficult. ${ }^{7}$ There are always likely to be multiple interventions within a large complex system, so attributing effects to a single intervention such as QI can be difficult. The case examples range from more intangible return from QI (such as revenue growth of the organisation, or staff engagement) where the link between QI and the outcome is less easy to define but broadly held to be true by those working in the system, to more concrete tangible return from QI (such as costs avoided or costs removed) where economic analysis is precise enough to estimate the number of pounds saved or removed due to the intervention. The framework could provide the basis for tracking and reporting on return to the board on a regular basis.

Calculating return on investment requires an evaluation of costs as well as benefits. The framework presented here is aimed at helping evaluate the benefits in a systematic way. Clearly there is also a need to calculate costs incurred, although that is not the focus of this paper. Costs of a complex large-scale intervention such as applying QI are as difficult to calculate as the benefits. While some costs, such as the costs of a central team or programme office, costs of training or costs of a strategic partner are fairly simple to understand, the bigger cost relates to the time invested by staff and other stakeholders across the system in learning and applying the method to improve services. This would be extremely hard to calculate, and for the purposes of evaluating return on investment, the board may be inclined to simply compare new tangible costs against benefits while excluding the intangible costs of time invested by staff across the organisation. This limitation should be noted, as there will be an opportunity cost to investing time into an endeavour such as QI.

\section{Patient, carer and family experience and outcomes}

The primary reason for applying QI within a healthcare organisation is to improve outcomes for those we serve, so this should be the main benefit that is realised from the work. At ELFT, every team is invited to consider what matters most to the staff working in the team, and the patients, service users, carers and families that receive care, and to use QI to solve the greatest quality issue. By focusing on what matters most to those closest to care - both receiving and delivering - we are attempting to design our QI work to be as personal and meaningful as possible.

Improving the experience and outcomes of the population we serve is the ultimate purpose of any healthcare provider, so this aspect of the business case for QI should align directly with the core purpose of the organisation. There will be countless case examples illustrating measurable improvements in patient, carer and family experience and outcomes in any organisation-wide application of QI. A few results from ELFT include the following (see charts in Supplementary file 1).

$>800 \%$ increase in self-directed community engagement in the low secure forensic service.

$>100 \%$ of patients having a discussion on the risks and benefits of psychotropics and future pregnancies prior to discharge from the ward.

> Improvement in percentage of inpatients agreeing or strongly agreeing that they were having a positive experience of the environment (from $52 \%$ to $70 \%$ ).

\section{Staff experience}

The QI process necessitates devolving power to teams to identify and solve their biggest issues, equipping them with tools and skills to tackle these systematically. This should, in theory, give staff more control over the system they work in, more autonomy to make changes and help them feel more engaged in their work. There is strong evidence that healthcare providers with happier, more engaged staff have better patient outcomes and improved financial performance. ${ }^{8}$ Figure 2 shows the ELFT scores (purple dot) for staff experience and engagement over the course of the trust's improvement journey, in comparison to the variation seen across all English healthcare providers. Overall staff engagement was highest across all 57 mental health and community trusts in 2016, and demonstrated the fourth consecutive year that ELFT has been in the top 5 . Staff feeling able to contribute towards improvements at work showed the top score across all NHS provider organisations in 2014 and 2016.

\section{Productivity and efficiency}

One of the goals of QI is to reduce unwanted variation and remove waste from the system, through supporting teams to focus on what matters to their service users. By removing nonvalue-adding steps in the process, and redesigning care around what adds value to the service user, teams are likely to see improvements in productivity and efficiency. These may free up time for teams and clinicians to focus on providing care, but may not have any effect on avoiding costs or removing costs. Figure 3 shows data from the ELFT clozapine clinic in City and Hackney, which has reduced variation and reduced overall time taken to return blood tests results to clinicians. This will help customers receive a faster and more predictable service, and allow the service to plan more effectively, but may have little effect on costs for the service.

Supplementary file 2 shows results from the ELFT collaborative learning system on improving access and flow in community teams, with 15 teams across a range of clinical services using QI to improve the process from referral to assessment and treatment. The teams have reduced the number of days people wait from referral to first appointment (by $20 \%$ over 2 years) and reduced non-attendance at first appointment (by $36 \%$ over 2 years), while seeing a $26 \%$ increase in number of referrals received during the same time period. This is likely to make teams more efficient and productive. In a block contract system, as for most mental health providers in England, this would have little discernible effect on cost or income. However, in tariff-based systems there are likely to be income gains from improving flow and throughput.

\section{Cost avoidance}

In some areas of QI work, improving the system may mean that we are able to avoid significant costs that were previously being incurred - either staff costs, or for equipment, materials or other overheads. Figure 4 shows data from the ELFT human resources team which has worked on improving the trust disciplinary process, reducing the average time from start to end of the process from an average of 112 days to an average of 55 days, a $51 \%$ reduction within 18 months.

As well as reducing anxiety for the person at the centre of the process, it also has the effect on reducing costs spent on paying 

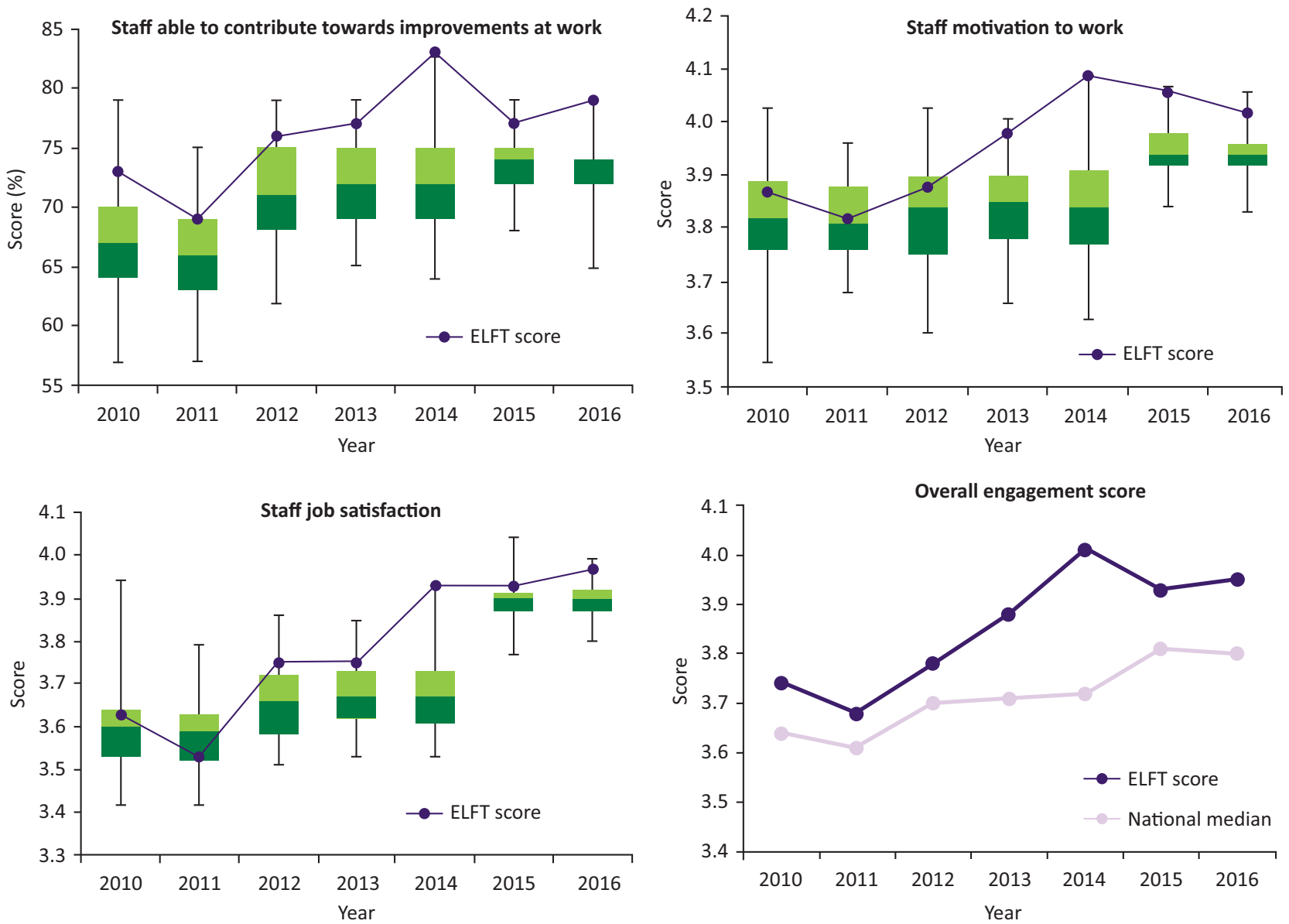

Fig 2. Staff experience and engagement at ELFT. Comparative data from the NHS Staff survey, showing ELFT scores in comparison with all other NHS provider organisations. ELFT = East London NHS Foundation Trust.

Fig 3. Data from the City \& Hackney clozapine service at ELFT showing time from request to results being received. ELFT $=$ East London NHS Foundation Trust; $\mathrm{LCL}=$ lower control limit; UCL = upper control limit.

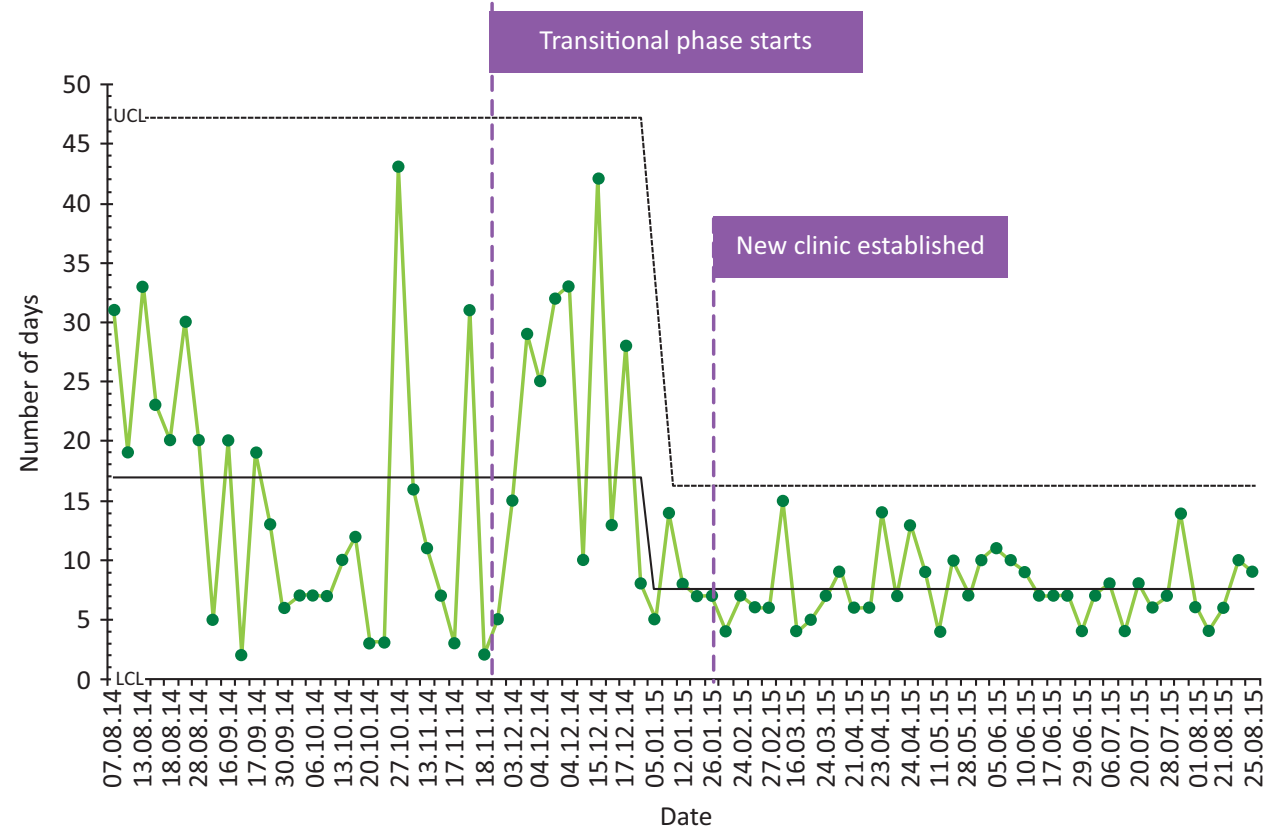




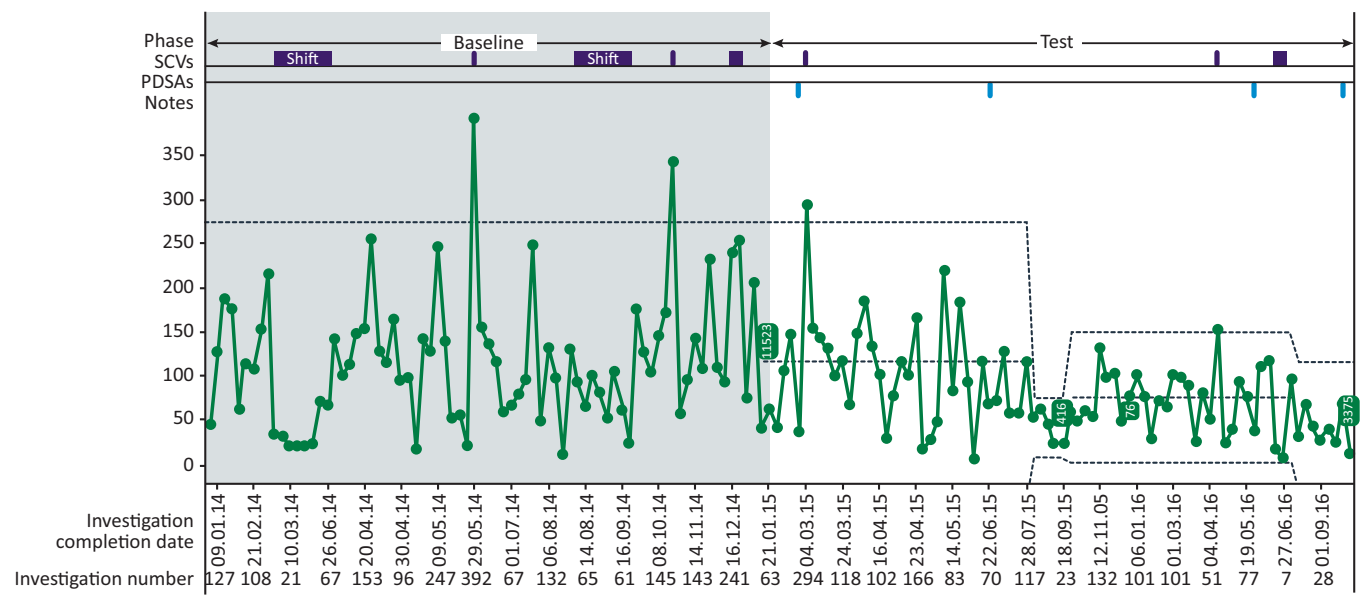

Fig 4. Number of days taken to complete the disciplinary process at ELFT. ELFT = East London NHS Foundation Trust: PDSAs = Plan-dostudy-act cycle (as test of change); SCV = special cause variation.

the staff member while they are suspended from work. The finance team at ELFT has calculated the cost of suspensions in 2014-15, and used this to estimate the cost per day of a staff member being suspended (see Table 1).

It is then possible to predict that if the average length of time for the disciplinary process can be reduced sustainably by 50 days (the team's data suggest they may even exceed this) and the number of cases does not change, this is like to lead to an annual cost avoidance of approximately $£ 430,000$.

In the following two case examples, ELFT has evaluated the cost avoided through reducing physical violence on acute adult mental health wards and older adult mental health wards. Both economic models involved finance teams working with clinicians to understand what happened when someone became violent on the ward, and evaluating the costs over the period of time related to violence (including medication costs, additional staff costs, repairs to the estate etc). The older adult case study demonstrated costs related to violence reducing from $£ 120,000$ over a 9-month period before the intervention, to $£ 60,000$ over a similar period after the intervention (Supplementary file 3, Table 2). A 36\% reduction in number of incidents of violence led to a $49 \%$ reduction in costs associated with violence. $^{9}$

A similar economic analysis of a reduction in physical violence on acute adult mental health wards showed that there was a saving of $£ 181,296$ attributable to a $40 \%$ reduction in violence over six wards (Supplementary files 4 and 5). ${ }^{10}$

Improving safety and staff experience at work is likely to lead to a reduction in staff absence from work, as the above two case

\begin{tabular}{|c|c|}
\hline \multicolumn{2}{|l|}{ Before (data for 2014-15) } \\
\hline Total cost & $£ 892,341$ \\
\hline Number of cases & 29 \\
\hline Average cost per case & $£ 30,770$ \\
\hline Average number of days suspended & 104 \\
\hline Average cost per day of suspension & $£ 296$ \\
\hline
\end{tabular}

examples have quantified. Reductions in staff sickness and use of temporary (bank or agency staff) as a result are both significant opportunities for cost avoidance. In addition, costs related to turnover of staff (recruitment, onboarding, temporary staffing to fill vacancies) can be avoided if staff remain with the employing organisation.

\section{Cost reduction}

In addition to avoiding costs, as in the case examples above, there may be opportunities for removing costs entirely from the system on a recurrent basis. This would directly support with the annual requirements for cost improvement that exist within the NHS for all provider organisations.

Figure 5 shows data from Ivory ward at ELFT which used QI to reduce bed occupancy on an older adult mental health ward from an average of $88 \%$ to a sustained new level of $58 \%$, by implementing a range of changes including better communication with liaison services in the acute hospital, daily review of patients, discharge planning huddles, visual display of the discharge process, stronger integration of social work within the discharge huddles and a discharge planning checklist. The team then spread these changes to Leadenhall, a similar ward in a different part of the organisation, and saw a reduction in bed occupancy from $77 \%$ to $51 \%$. With both wards operating nearly half full, it has then been possible to move to just one ward, with the opportunity to close one ward. This will deliver a cost reduction of approximately $£ 1$ million per year. In the English health economy where provider trusts have to find ways each year to reduce their bottom line by

Table 2. Economic evaluation of costs attributable to violence prior and after the QI project

The 6 months The 6 months after prior to the the QI project QI project completed

Number of incidents $\quad 44 \quad 28$

Costs attributable to $£ 119,988 \quad £ 61,376$ violence

QI = quality improvement 

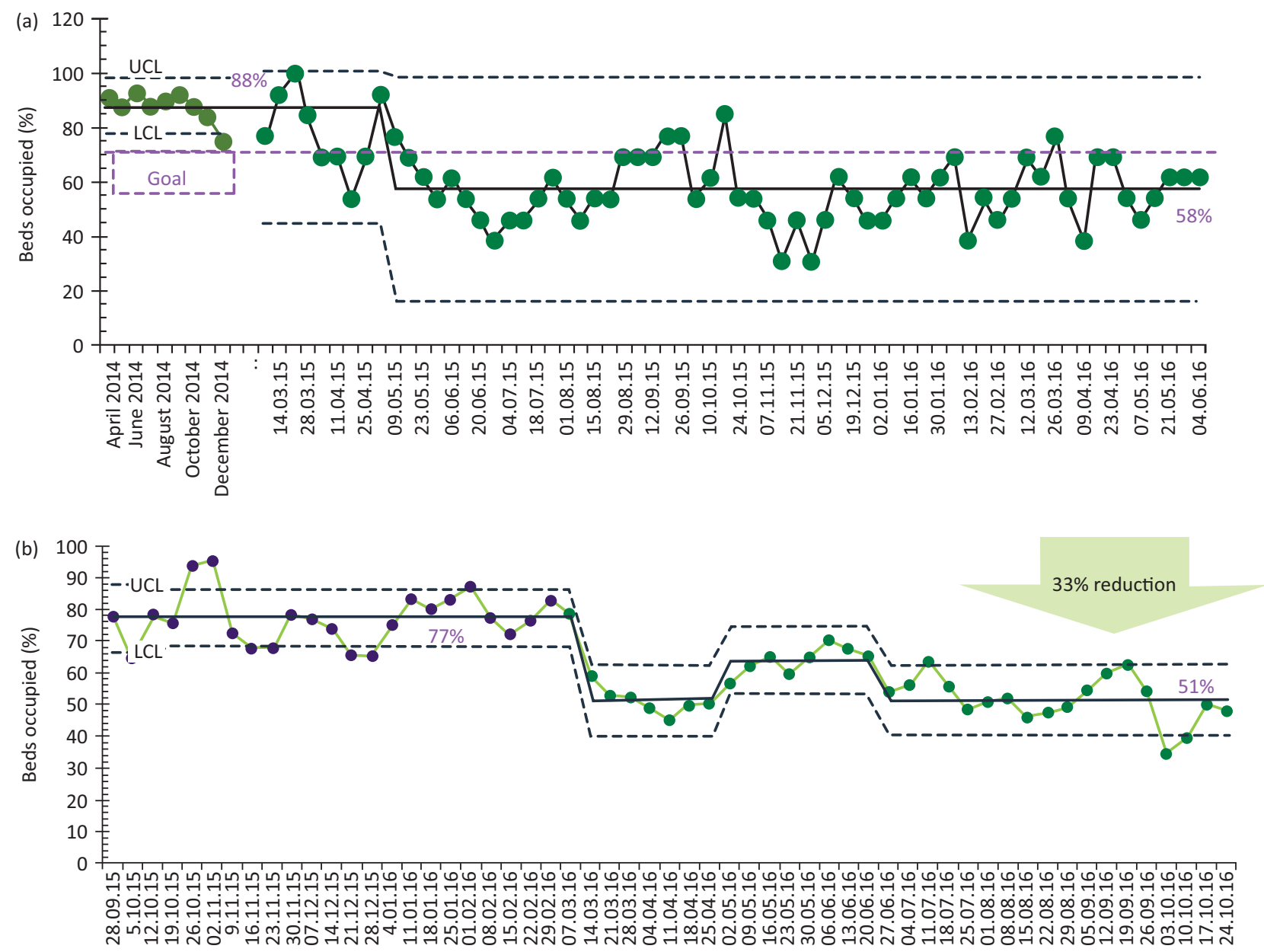

Fig 5. Reduction in bed occupancy on two older adult mental health wards through QI. (a) Ivory ward (excluding leave), (b) Leadenhall ward. QI = quality improvement.

2-3\%, this kind of QI-realised cost reduction can help significantly with other cost improvement efforts.

New work has just begun at ELFT aimed at cost reduction, through targeting opportunities to reduce non-pay costs in salary overpayments, translation and interpreter costs, and postage/ mailing.

\section{Revenue}

Applying QI at scale brings new strategic capability within an organisation, adding competitive advantage within the market. This has the potential to realise the benefit of attracting revenue, including through acquiring new business based on reputation for quality and improvement. Figure 6 shows how ELFT's revenue has increased over the last few years. While it is not possible to draw any causal link between the application of QI and organisational revenue, a focus on quality, being able to demonstrate improved outcomes through QI and a reputation for being a quality leader are believed to have contributed to the acquisition of new business.

In addition, the discipline of continuous improvement and using data more rigorously to focus on what matters most to service users means that services will be able to better demonstrate their value. This reduces the risk of being decommissioned or losing a service to a competing organisation, thereby retaining revenue for the organisation.

Finally, an expertise in QI opens the opportunity to support other organisations with the same endeavour. ELFT generates a small

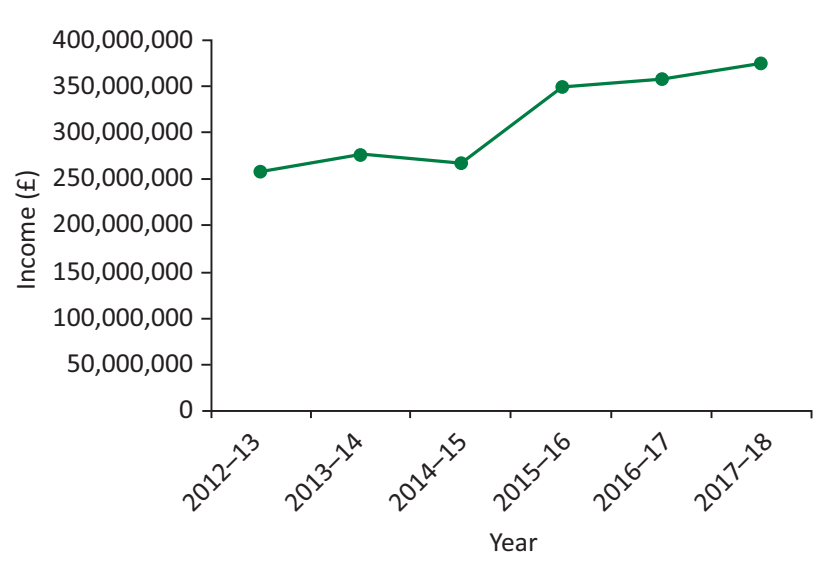

Fig 6. ELFT annual income over the 6 years from 2012-13 and 2017-18. ELFT = East London NHS Foundation Trust. 
amount of revenue through expert faculty who deliver training and coach other organisations in their QI journey.

The work contained in this article has not required formal ethics approval as it falls within the remit of service improvement work and is governed by the structures in place to manage formal quality improvement projects at East London NHS Foundation Trust.

\section{Summary}

A key message to those contemplating undertaking large-scale application of QI in an organisation is to consider the return on this investment at multiple levels. Clearly, the primary driver for this strategic decision is to improve outcomes and experience for those that receive care and services. However, the organisation will also reap dividend from enhanced engagement and motivation of staff, improved productivity and efficiency of teams, cost avoidance (reducing cost pressures), cost reduction and the possibility of increased revenue. All of these potential avenues for return on investment should be evaluated and considered as part of the decision on whether to invest in QI, and in evaluating the impact of applying QI at scale within an organisation.

This paper has proposed a framework for evaluating return on investment from QI within the health economy of the English NHS, using East London NHS Foundation Trust's QI programme as a case example. The framework could equally be applied to any large-scale QI endeavour within other organisations, both within health and social care or in other industries. For any organisation or large system, in relation to the application of QI, it should be possible to evaluate direct benefit to the customer, improvement in experience at work for employees, improvement in productivity and efficiency, costs avoided, costs removed and increase in business revenue. This would form the basis of calculating benefit and thereafter return on investment from QI. Utilising this framework should help articulate to key stakeholders that the return on investment from QI cannot solely be evaluated in terms of costs removed from the system, even though this may be a pressing need in many health economies. Recognising the multiple levels of return can also help better link the application of QI to the core strategic priorities of the organisation, through evaluating impact on patient outcomes and experience, and the experience of staff.

\section{Key points}

> Return on investment from QI can be understood and evaluated at multiple levels

$>$ Return from QI is first and foremost about improving outcomes for the population served, secondly about improving the experience of those delivering care, and thirdly about becoming more efficient, removing waste, reducing cost and increasing revenue

> The ELFT framework for evaluating return on investment might provide a way to capture and report on return on investment within organisation undertaking large-scale strategic quality improvement initiatives.

\section{Supplementary material}

Additional supplementary material may be found in the online version of this article at http://futurehospital.rcpjournal.org

S1 - Three projects from ELFT demonstrating improvement in outcomes for service users

S2 - Results from the ELFT learning system on improving access and flow in community teams

S3 - Outcome data for violence reduction work on three older adult wards

S4 - Violence reduction across six acute adult wards at ELFT

S5 - Economic evaluation of cost impact of reducing violence on acute adult ward

\section{References}

1 Ham C, Berwick D, Dixon J. Improving quality in the English NHS: A strategy for action. The King's Fund, 2016.

2 Collins J. Good to Great: Why some companies make the leap... and others don't, 1st edn. HarperBusiness, 2001.

3 Swensen S, Dilling J, Carty P, Bolton J, Harper C. The Business Case for Healthcare Quality Improvement. J Patient Saf 2013:9:44-52.

4 Ross S, Naylor C. Quality improvement in mental health. The King's Fund, 2017.

5 Nolan TW. Execution of strategic improvement initiatives to produce system-level results. IHI Innovation Series white paper. Cambridge, MA: IHI, 2007.

6 Institute for Healthcare Improvement. Building a culture of Improvement at East London NHS Foundation Trust. Cambridge, MA: IHI, 2016.

7 Dixon-Woods M, Martin G. Does quality improvement improve quality? Future Hosp ] 2016;3:191-4.

8 West M, Dawson J. Employee engagement and NHS Performance. The King's Fund, 2012.

9 Brown J, Fawzi W, McCarthy C et al. Safer Wards: reducing violence on older people's mental health wards. BMJ Qual Improv Rep 2015;4:pii: u207447.w2977

10 Taylor-Watt ], Cruickshank A, Innes ], Brome B, Shah A. Reducing physical violence and developing a safety culture across wards in East London. British Journal of mental health nursing 2017;6:3543.

Address for correspondence: Dr Amar Shah, East London NHS Foundation Trust, 9 Alie Street, London E1 8DE, UK.

Email: amarshah@nhs.net 